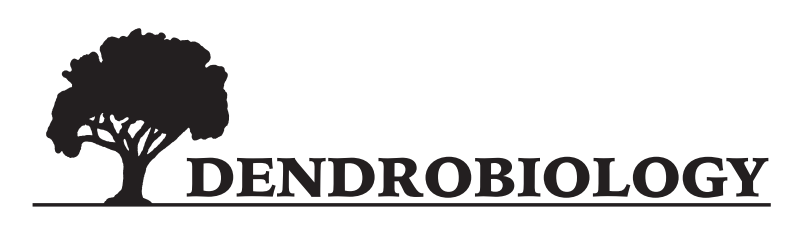

2018, vol. 79, 34-46

http://dx.doi.org/10.12657/denbio.079.004

\author{
Szymon Jastrzębowski, Krzysztof Ukalski, Marcin Klisz, \\ Joanna Ukalska, Pawet Przybylski, Jan Matras, Władystaw Barzdajn, \\ Wojciech Kowalkowski
}

\title{
Assessment of the height stability in progeny of Fagus sylvatica L. populations using the GGE biplot method
}

Received: 23 June 2017; Accepted: 27 October 2017

\begin{abstract}
Forecasted climate changes demand selection of populations (seed stands) and genotypes (plus trees) best adapted to changing environmental conditions and displaying limited genotype $\times$ environment $(\mathrm{G} \times \mathrm{E})$ interaction. Analysis of multi-environment trials (METs) allows to recognize differences between populations and environments, as well as $\mathrm{G} \times \mathrm{E}$ interaction. To define stability of tree height we used a GGE biplot graphic method based on the results of measurement of 5- and 10-year-old trees originating from 30 European beech populations tested at three experimental sites. Majority of variance was explained in terms of the impact of environment. The studied environments were characterised by a similar discriminating ability and representativeness of growth conditions. Two mega-environments were identified as the studied populations of beech differed in their adaptation to local growth conditions. The analysed set of populations included those growing particularly well under the specific environmental conditions, and others displaying more general adaptability. The GGE biplot method is useful in breeding of forest trees.
\end{abstract}

Keywords: $\mathrm{G} \times$ E interaction, GGE biplot, MET, adaptation, European beech

Addresses: Sz. Jastrzębowski - Department of Silviculture and Forest Tree Genetics, Forest Research Institute, Braci Leśnej Street, No 3, Sękocin Stary, 05-090 Raszyn, Poland, s.jastrzebowski@ibles.waw.pl K. Ukalski - Biometry Division, Department of Econometrics and Statistics, Faculty of Applied Informatics and Mathematics, Warsaw University of Life Sciences Ul. Nowoursynowska 159 02-776 Warszawa, krzysztof_ukalski@sggw.pl

M. Klisz - Department of Silviculture and Forest Tree Genetics, Forest Research Institute, Braci Leśnej Street, No 3, Sękocin Stary, 05-090 Raszyn, Poland, m.klisz@ibles.waw.pl

J. Ukalska - Biometry Division, Department of Econometrics and Statistics, Faculty of Applied Informatics and Mathematics, Warsaw University of Life Sciences Ul. Nowoursynowska 159 02-776 Warszawa, joanna_ukalska@sggw.pl

P. Przybylski - Department of Silviculture and Forest Tree Genetics, Forest Research Institute, Braci Leśnej Street, No 3, Sękocin Stary, 05-090 Raszyn, Poland, p.przybylski@ibles.waw.pl

J. Matras - Department of Silviculture and Forest Tree Genetics, Forest Research Institute, Braci Leśnej Street, No 3, Sękocin Stary, 05-090 Raszyn, Poland, j.matras@ibles.waw.pl

W. Barzdajn - Department of Silviculture, Faculty of Forestry, Poznań University of Life Science, Wojska Polskiego 69, 60-625 Poznań, Poland, barzdajn@up.poznan.pl

W. Kowalkowski - Department of Silviculture, Faculty of Forestry, Poznań University of Life Science, Wojska Polskiego 69, 60-625 Poznań, Poland, wojkowal@up.poznan.pl 


\section{Introduction}

The European beech (Fagus sylvatica L.) is a key forest-forming broadleaved tree species in Poland, where it currently covers $530,641.6$ ha of forest, i.e. $5.8 \%$ of the total forest area managed by the State Forests, and $22.6 \%$ of forest area in National Parks (ca 41,769.72 ha). However, data on the presence and structure of forest habitats in Poland suggest further possibilities to increase the role of broadleaved tree species, including beech, in the country's forests (Raport o lasach, 2014). The seed base of beech currently includes 122 selected seed stands (over 2009.3 ha), 604 maternal (plus) trees, 5 clonal seed orchards (35 ha) and 8 seedling seed orchards (43 ha). Fifteen seed regions for the species have been designated in Poland, with 5 of them located in the mountains or uplands (Fonder et al., 2007). The European beech reaches the eastern limit of its natural distribution range in Poland, with two distinguished centres of occurrence - Pomeranian and montane/upland (Boratyńska \& Boratyński, 1990), where ecological requirements for growth of beech in natural communities are met (Dzwonko, 1990). However, according to Tarasiuk (1992), the entire country represents a potential territory for European beech to play a more prominent role in managed stands.

In line with the need to make future forest stands more stable, healthy and productive, a detailed study of beech seed stands is being conducted, with the aim of recognizing their genetic value, and ensuring adequate and effective preservation. To better recognize variation among Polish beech populations, many study sites have been established since 1995, with research there seeking best options for beech cultivation under various environmental conditions (Sabor, 1999). Work done elsewhere, especially in Germany and France, points that populations within this species indeed show marked variation in both growth and qualitative traits (Teissier du Cros, 1977; von Wüehlisch, 2004; Ivanković et al., 2008). However, many studies also indicate the presence of strong $\mathrm{G} \times \mathrm{E}$ interaction (Krahl-Urban, 1958; von Wüehlisch et al., 2008), and ecotypic variation. It is therefore clear that transfer of beech seeds over long distances may be not beneficial in terms of commercial results.

Breeding of forest trees needs to encompass not only an effort to improve quantitative and qualitative traits, but also work to assess the stability of those traits, both between different environments and throughout a period of many years (Rodrigues et al., 2008). Predicted climatic change necessitates artificial selection for the populations and genotypes faring best when it comes to adaptation to changing environmental conditions, but at the same time showing a limited $\mathrm{G} \times \mathrm{E}$ interaction. The $\mathrm{G} \times \mathrm{E}$ interaction is of influence where assessments of heritability are concerned, as well as decisions about silvicultural objectives and practices (Yan \& Kang, 2002). The G×E interaction is in fact a key parameter for plant-breeders in general (Kang, 1990; Kang \& Gauch, 1996; Annicchiarico, 2002). Breeding strategies for forest trees are often based on a search for populations with low values of the $G \times E$ interaction, which can thus be described as plastic (Giertych, 1982). With only the results of short-term experiments available, it is hard to to gain at unambiguous recommendations in line with which practical decisions may be taken. However, results obtained after 5 and 10 years of growth do allow to analyse the adaptation and productivity that is represented by tree height. The determination of the stability of this trait in the analysed populations is inter alia of key importance to the development of policy on transfers of seeds (Yeiser et al., 2001).

Where work based on multi-environment trials (MET) aims to depict the G $\times E$ interaction, as well as its relationship with $G$ (i.e. the effect of genotype) as such, methods based on analysis of variance and regression analysis are found to be less effective than multidimensional methods (Crossa, 1990). A biplot of the GGE type facilitates presentation of relevant relationships in form that is clear and easy to interpret. The GGE effect comprises $\mathrm{G}$ - the effect of genotypes, as well as $\mathrm{G} \times \mathrm{E}$ - that is due to the interaction between genotype and the environment (Yan et al., 2000). The GGE biplots are generated by reference to the values of the first two components in the Principal Component Analysis (PCA), i.e. PC1 and PC2. With the aid of GGE biplots it becomes possible to establish correlations between environments, to define the existence of mega-environments, and to estimate the real productivity of genotypes within these mega-enviroments (DeLacy et al., 1996; Ding et al., 2008). From the practical point of view genotypes that can be recommended for growing in given environments are identified (Gauch \& Zobel, 1997; Yan et al., 2000; Yan et al., 2001; Ukalski et al., 2010a; Ukalski et al., 2010b; Ukalski \& Klisz, 2016; Klisz et al., 2017). One option is to select those genotypes that show favorable and stable values for the traits of interest in the so-called average environment (Ukalski \& Klisz, 2016), as identified with the AEC or Average Environment Coordination Method (Yan \& Hunt, 2001; Yan, 2002). Yet a further possibility is to seek out genotypes that approximate the theoretical "ideal" genotype, i.e. the one associated with both favorable and stable values for a given trait (or traits) in all of the studied environments (Yan et al., 2000; Yan \& Rajcan, 2002).

The "Program of testing the progeny of selected seed stands, plus trees, clonal and seedling seed orchards" in the State Forest aims to determine the genetic value and silvicultural properties of the forest basic material which is used in forestry practice. 
It further aims to prepare the recommendations for the rational use of seed base (forest basic material) by determining the directions of possible transfer of forest reproductive material (Program, 2011). Stability of growth traits in various conditions is very important in selection of populations in the case of environmental change. The purpose of this study was to determine stability of tree height growth among progeny of European beech populations originating from southern Poland and growing in montane and upland conditions, using the GGE biplot analysis.

\section{Materials and Methods}

\section{Sites and experimental design}

The study includes progeny of 30 selected seed stands (populations) of European beech from southern Poland. In 2004 seeds were collected from 50 trees chosen randomly along a transect in each population. Minimal distance between trees in a transect was $20-25 \mathrm{~m}$. Seeds were collected from healthy trees in the year of a good seed crop. All seeds were stored at the Kostrzyca Forest Gene Bank until the time of sowing in a nursery in spring 2005. Seedlings were raised in containers in the Gidle Forest Nursery $\left(50^{\circ} 54^{\prime} \mathrm{N}, 19^{\circ} 37^{\prime} \mathrm{E}\right), \sim 180 \mathrm{~km}$ north of the experimental sites. After one year of growing in the nursery seedlings were transferred directly to the experimental sites in spring 2006.

Three trial plots were estabilished in southern and south-western Poland - in Sucha Beskidzka (SB) (N49³7'40" E19³2'48”, 825 m a.s.l.), Lądek Zdrój (LZ) (N50¹9'50" E16 45'03", 520 m a.s.l.) and Zlotoryja (ZL) (N51 ${ }^{\circ} 56^{\prime} 59^{\prime \prime}$ E15 48'53”, 400 $\mathrm{m}$ a.s.l.) - Fig. 1. At each site the trial was set up in a complete block design with four replications (blocks). Each tested population was represented by 400 planted seedlings (100 in each replications) planted in $1.5 \times 1.5 \mathrm{~m}$ spacing.

Habitat conditions associated with these study areas were diverse. According to the Polish forest typology the SB site was a montane broadleaved forest $(L G)$; the $\mathrm{ZL}$ site was a montane mixed broadleaved forest $(L M G)$, and the LZ site was a fresh montane mixed broadleaved forest (LMGsw). The first height measurements on these plots were made in 2010, and the second measurements were done five years later, in 2015.

\section{Statistical analysis}

Analysis of variance was used to study the differentiation in tree heights among the studied populations, as well as the influence on this differentiation exerted by different environments. The linear mixed model with environment, population and the $\mathrm{G} \times \mathrm{E}$ interaction as class variables (Piepho, 1997) was applied, using the equation:

$$
y_{i j k}=\mu+r_{k}\left(e_{j}\right)+g_{i}+e_{j}+g e_{i j}+\varepsilon_{i j k}
$$

where: $y_{i j k}$ is the trait value observed for the $i$-th genotype $(i=1, \ldots, 30)$ in the $j$-th environment $(j=1, \ldots, 3)$ in the $k$-th block, $\mu$ is the overall mean, $g_{i}$ is the fixed effect of the $i$-th genotype, $e_{j}$ is the random effect of the $j$-th environment, $g e_{i j}$ is the random effect of the genotype $\times$ environment interaction, $r_{k}\left(e_{j}\right)$ is the random effect of the $k$-th block in the $j$-th environment $(k=1, \ldots, 4)$, and $\varepsilon_{i j k}$ is the experimental error. The genotype effect in the model (1) was treated as fixed, due to the fact that the tested genotypes were selected decisively with reference to analyzed traits. The environment effect was treated as random because experimental sites represented the widest possible spectrum of environmental conditions for a given species (Saxton, 2004). The F statistic values for the genotype and environment effects in the model (1) were determined using Hocking's approach (Hocking \& Speed, 1975).

To perform the biplot analysis, the two-dimensional table of means should be factorized using singular value decomposition (SVD) (Golub \& Reinsch, 1971), or equivalently, calculations can be performed using PCA (Ukalski \& Klisz, 2016). We used PCA on the genotypic means across environments (Table 1). This offered a basis for identifying the most representative environment. The discriminating potential of the environments was determined, and the 30 populations of beech assessed in relation to values of tree height and their stability.

The AEC method was used to determine the similarity of environments depicted on the GGE biplot. The average environment was identified by reference to principal components $\mathrm{PC} 1$ and $\mathrm{PC} 2$ as related to the three environments. An AE axis was run though the AE point and the centre of the coordinate system. Similarities between all possible pairs of sites were determined using two methods. The first related to $\alpha_{i j}$ angles between vectors $\mathrm{OA}_{\mathrm{i}}$ and $\mathrm{OB}_{\mathrm{j}}$ (where $\mathrm{OA}_{\mathrm{i}}$ and $\mathrm{OB}_{\mathrm{j}}$ are the lengths of the vectors between the origin of the coordinate system and sites A and B, respectively). The second method involved Pearson correlation coefficients calculated for all pairs of environments on the basis of PCA values.

Populations mean height-related performance were described by the their position in relation to average environment (AE) axis on the GGE biplots. The zero value for $\mathrm{AE}$ denotes the mean value for traits through the environments. Populations with high values for $\mathrm{AE}$ are characterised by high values of tree height. The length of the line segment between the population location and its orthogonal projection 

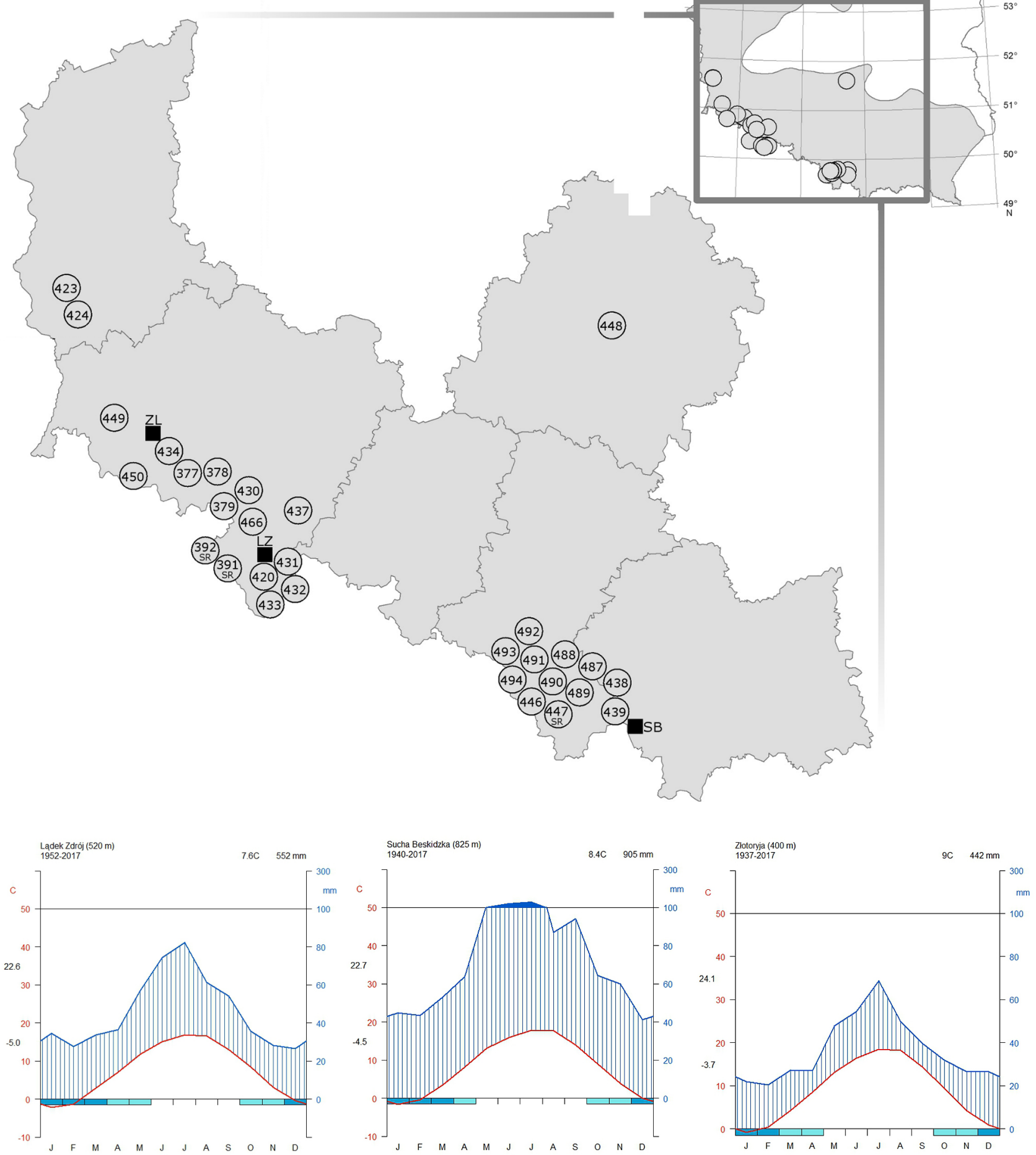

Fig. 1. Location and climatic conditions of study sites. Bottom panel: plots of the precipitation and temperature monthly averages, annual averages of both elements (in the upper part) and monthly averages of the daily maximum and minimum temperatures of the warmest and coldest months respectively (at the left margin); rectangle under the $0^{\circ} \mathrm{C}$ axis frost likelihood (darker blue when the average daily minimum is zero or negative, lighter blue when absolute monthly minimum is zero or negative); blue vertical pattern depicts the humid months. Upper panel: Squares relate to trial sites (environments); circles with numbers (420, 377 etc.) - tested populations. Grey line - boundaries of Forest Districts 
Table 1. Means and variation coefficients of height [cm] and survival [\%] of tested populations, 5 and 10 years after planting, SB - Sucha Beskidzka, LZ - Lądek Zdrój, ZL - Złotoryja

\begin{tabular}{|c|c|c|c|c|c|c|c|c|c|c|c|c|c|c|c|c|c|}
\hline \multirow{3}{*}{$\begin{array}{c}\text { Population } \\
\text { no. }\end{array}$} & \multirow{3}{*}{$\begin{array}{l}\text { Population } \\
\text { name }\end{array}$} & \multicolumn{8}{|c|}{ Mean height $[\mathrm{cm}](\mathrm{CV}[\%])$} & \multicolumn{8}{|c|}{ Mean survival [\%] } \\
\hline & & \multicolumn{2}{|c|}{ SB } & \multicolumn{2}{|c|}{$\mathrm{LZ}$} & \multicolumn{2}{|c|}{ ZL } & \multicolumn{2}{|c|}{$\begin{array}{c}\text { Mean } \\
\text { across sites }\end{array}$} & \multicolumn{2}{|c|}{ SB } & \multicolumn{2}{|c|}{$\mathrm{LZ}$} & \multicolumn{2}{|c|}{$\mathrm{ZL}$} & \multicolumn{2}{|c|}{$\begin{array}{c}\text { Mean } \\
\text { across sites }\end{array}$} \\
\hline & & 2010 & 2015 & 2010 & 2015 & 2010 & 2015 & 2010 & 2015 & 2010 & 2015 & 2010 & 2015 & 2010 & 2015 & 2010 & 2015 \\
\hline 377 & Wałbrzych & $\begin{array}{l}131 \\
(8)\end{array}$ & $\begin{array}{l}430 \\
(26)\end{array}$ & $\begin{array}{l}177 \\
(6)\end{array}$ & $\begin{array}{c}480 \\
(6)\end{array}$ & $\begin{array}{c}166 \\
(8)\end{array}$ & $\begin{array}{l}490^{f} \\
(21)\end{array}$ & $\begin{array}{l}160 \\
(13)\end{array}$ & $\begin{array}{l}455 \\
(22)\end{array}$ & 54 & 49 & 97 & 89 & 92 & 66 & 81 & 68 \\
\hline 378 & Wałbrzych & $\begin{array}{l}124 \\
(11)\end{array}$ & $\begin{array}{l}440^{f} \\
(15)\end{array}$ & $\begin{array}{l}179 \\
(4)\end{array}$ & $\begin{array}{c}510^{f} \\
(6)\end{array}$ & $\begin{array}{l}174 \\
(15)\end{array}$ & $\begin{array}{l}470 \\
(17)\end{array}$ & $\begin{array}{l}159 \\
(18)\end{array}$ & $\begin{array}{l}470 \\
(21)\end{array}$ & 76 & 67 & 96 & 89 & 94 & 78 & 89 & 78 \\
\hline 379 & Wałbrzych & $\begin{array}{l}114 \\
(13)\end{array}$ & $\begin{array}{l}400 \\
(29)\end{array}$ & $\begin{array}{l}169 \\
(8)\end{array}$ & $\begin{array}{l}480 \\
(10)\end{array}$ & $\begin{array}{l}143 \\
(25)\end{array}$ & $\begin{array}{l}400 \\
(21)\end{array}$ & $\begin{array}{l}142 \\
(21)\end{array}$ & $\begin{array}{l}427 \\
(25)\end{array}$ & 67 & 56 & 95 & 90 & 97 & 90 & 86 & 79 \\
\hline 391 & Zdroje & $\begin{array}{l}121 \\
(10)\end{array}$ & $\begin{array}{l}400 \\
(12)\end{array}$ & $\begin{array}{l}156 \\
(8)\end{array}$ & $\begin{array}{l}450 \\
(9)\end{array}$ & $\begin{array}{l}146 \\
(15)\end{array}$ & $\begin{array}{l}430 \\
(16)\end{array}$ & $\begin{array}{l}141 \\
(14)\end{array}$ & $\begin{array}{l}427 \\
(15)\end{array}$ & 86 & 73 & 89 & 79 & 81 & 63 & 85 & 72 \\
\hline 392 & Zdroje & $\begin{array}{l}121 \\
(10)\end{array}$ & $\begin{array}{l}430^{f} \\
(31)\end{array}$ & $\begin{array}{l}171 \\
(3)\end{array}$ & $\begin{array}{l}500 f \\
(13)\end{array}$ & $\begin{array}{l}138 \\
(5)\end{array}$ & $\begin{array}{l}470^{f} \\
(19)\end{array}$ & $\begin{array}{l}143 \\
(13)\end{array}$ & $\begin{array}{l}467 \\
(27)\end{array}$ & 62 & 56 & 91 & 89 & 92 & 76 & 82 & 74 \\
\hline 420 & Międzylesie & $\begin{array}{l}105 \\
(18)\end{array}$ & $\begin{array}{l}420^{f} \\
(32)\end{array}$ & $\begin{array}{l}157 \\
(5)\end{array}$ & $\begin{array}{l}440 \\
(11)\end{array}$ & $\begin{array}{l}158 \\
(11)\end{array}$ & $\begin{array}{l}510^{f} \\
(16)\end{array}$ & $\begin{array}{l}140 \\
(19)\end{array}$ & $\begin{array}{l}440 \\
(27)\end{array}$ & 58 & 45 & 91 & 84 & 96 & 87 & 82 & 72 \\
\hline 423 & Lipinki & $\begin{array}{l}125 \\
(7)\end{array}$ & $\begin{array}{l}400 \\
(30)\end{array}$ & $\begin{array}{l}152 \\
(7)\end{array}$ & $\begin{array}{l}430 \\
(14)\end{array}$ & $\begin{array}{l}158 \\
(17)\end{array}$ & $\begin{array}{l}470 \\
(20)\end{array}$ & $\begin{array}{l}145 \\
(15)\end{array}$ & $\begin{array}{l}433 \\
(24)\end{array}$ & 65 & 61 & 95 & 86 & 86 & 65 & 82 & 71 \\
\hline 424 & Lipinki & $\begin{array}{l}126 \\
(20)\end{array}$ & $\begin{array}{l}410 \\
(13)\end{array}$ & $\begin{array}{l}158 \\
(3)\end{array}$ & $\begin{array}{c}500^{f} \\
(4)\end{array}$ & $\begin{array}{l}134 \\
(20)\end{array}$ & $\begin{array}{c}380 \\
(8)\end{array}$ & $\begin{array}{l}139 \\
(16)\end{array}$ & $\begin{array}{l}395 \\
(18)\end{array}$ & 73 & 65 & 95 & 89 & 90 & 78 & 86 & 77 \\
\hline 430 & Świdnica & $\begin{array}{l}112 \\
(12)\end{array}$ & $\begin{array}{l}340^{\mathrm{s}} \\
(23)\end{array}$ & $\begin{array}{l}162 \\
(12)\end{array}$ & $\begin{array}{l}390^{\mathrm{s}} \\
(10)\end{array}$ & $\begin{array}{l}136 \\
(18)\end{array}$ & $\begin{array}{l}360^{s} \\
(16)\end{array}$ & $\begin{array}{l}137 \\
(19)\end{array}$ & $\begin{array}{l}363 \\
(18)\end{array}$ & 65 & 56 & 94 & 89 & 96 & 87 & 85 & 77 \\
\hline 431 & Lądek Zdrój & $\begin{array}{l}119 \\
(12)\end{array}$ & $\begin{array}{l}430^{f} \\
(26)\end{array}$ & $\begin{array}{l}155 \\
(8)\end{array}$ & $\begin{array}{l}430 \\
(8)\end{array}$ & $\begin{array}{l}150 \\
(9)\end{array}$ & $\begin{array}{l}480^{f} \\
(16)\end{array}$ & $\begin{array}{l}141 \\
(14)\end{array}$ & $\begin{array}{l}430 \\
(22)\end{array}$ & 69 & 62 & 95 & 85 & 96 & 66 & 87 & 71 \\
\hline 432 & Lądek Zdrój & $\begin{array}{l}123 \\
(8)\end{array}$ & $\begin{array}{l}420 \\
(16)\end{array}$ & $\begin{array}{l}175 \\
(13)\end{array}$ & $\begin{array}{l}480 \\
(16)\end{array}$ & $\begin{array}{l}134 \\
(19)\end{array}$ & $\begin{array}{l}400 \\
(19)\end{array}$ & $\begin{array}{l}144 \\
(18)\end{array}$ & $\begin{array}{l}433 \\
(20)\end{array}$ & 74 & 69 & 94 & 83 & 90 & 72 & 86 & 75 \\
\hline 433 & Lądek Zdrój & $\begin{array}{l}122 \\
(10)\end{array}$ & $\begin{array}{l}400 \\
(21)\end{array}$ & $\begin{array}{l}163 \\
(10)\end{array}$ & $\begin{array}{l}451 \\
(11)\end{array}$ & $\begin{array}{l}159 \\
(26)\end{array}$ & $\begin{array}{l}430 \\
(36)\end{array}$ & $\begin{array}{l}148 \\
(22)\end{array}$ & $\begin{array}{l}427 \\
(28)\end{array}$ & 73 & 63 & 93 & 90 & 96 & 91 & 87 & 81 \\
\hline 434 & Jawor & $\begin{array}{l}118 \\
(8)\end{array}$ & $\begin{array}{l}390 \\
(17)\end{array}$ & $\begin{array}{l}165 \\
(16)\end{array}$ & $\begin{array}{l}410^{\mathrm{s}} \\
(23)\end{array}$ & $\begin{array}{l}158 \\
(14)\end{array}$ & $\begin{array}{l}440 \\
(22)\end{array}$ & $\begin{array}{l}147 \\
(18)\end{array}$ & $\begin{array}{l}415 \\
(27)\end{array}$ & 66 & 58 & 94 & 88 & 88 & 66 & 82 & 71 \\
\hline 437 & Henryków & $\begin{array}{l}130 \\
(12)\end{array}$ & $\begin{array}{l}410 \\
(18)\end{array}$ & $\begin{array}{l}178 \\
(11)\end{array}$ & $\begin{array}{l}510^{f} \\
(11)\end{array}$ & $\begin{array}{l}120 \\
(30)\end{array}$ & $\begin{array}{l}380 \\
(25)\end{array}$ & $\begin{array}{l}143 \\
(24)\end{array}$ & $\begin{array}{l}395 \\
(24)\end{array}$ & 71 & 63 & 94 & 58 & 88 & 61 & 84 & 61 \\
\hline 438 & Sucha & $\begin{array}{c}98 \\
(21)\end{array}$ & $\begin{array}{l}330^{s} \\
(30)\end{array}$ & $\begin{array}{l}144 \\
(16)\end{array}$ & $\begin{array}{c}420^{\mathrm{s}} \\
(6)\end{array}$ & $\begin{array}{c}97 \\
(15)\end{array}$ & $\begin{array}{l}320^{s} \\
(14)\end{array}$ & $\begin{array}{l}113 \\
(24)\end{array}$ & $\begin{array}{l}420 \\
(27)\end{array}$ & 50 & 35 & 83 & 43 & 96 & 63 & 76 & 47 \\
\hline 439 & Sucha & $\begin{array}{l}126 \\
(8)\end{array}$ & $\begin{array}{l}430 \\
(33)\end{array}$ & $\begin{array}{l}178 \\
(8)\end{array}$ & $\begin{array}{l}500 \\
(19)\end{array}$ & $\begin{array}{l}154 \\
(9)\end{array}$ & $\begin{array}{l}480^{f} \\
(17)\end{array}$ & $\begin{array}{l}153 \\
(14)\end{array}$ & $\begin{array}{l}465 \\
(28)\end{array}$ & 70 & 64 & 97 & 89 & 91 & 65 & 86 & 73 \\
\hline 446 & Ustroń & $\begin{array}{l}125 \\
(21)\end{array}$ & $\begin{array}{l}420 \\
(26)\end{array}$ & $\begin{array}{l}165 \\
(5)\end{array}$ & $\begin{array}{l}520^{f} \\
(14)\end{array}$ & $\begin{array}{l}151 \\
(14)\end{array}$ & $\begin{array}{l}430 \\
(10)\end{array}$ & $\begin{array}{l}147 \\
\text { (17) }\end{array}$ & $\begin{array}{l}425 \\
(24)\end{array}$ & 71 & 63 & 95 & 89 & 92 & 77 & 86 & 76 \\
\hline 447 & Ustroń & $\begin{array}{l}113 \\
(11)\end{array}$ & $\begin{array}{l}380 \\
(31)\end{array}$ & $\begin{array}{l}165 \\
(11)\end{array}$ & $\begin{array}{l}450 \\
(14)\end{array}$ & $\begin{array}{l}151 \\
\text { (20) }\end{array}$ & $\begin{array}{l}400 \\
(32)\end{array}$ & $\begin{array}{l}143 \\
(20)\end{array}$ & $\begin{array}{l}410 \\
(29)\end{array}$ & 69 & 58 & 93 & 88 & 96 & 91 & 86 & 79 \\
\hline 448 & Kolumna & $\begin{array}{l}115 \\
(12)\end{array}$ & $\begin{array}{l}390 \\
(28)\end{array}$ & $\begin{array}{l}154 \\
(12)\end{array}$ & $\begin{array}{l}480 \\
(11)\end{array}$ & $\begin{array}{l}116 \\
(25)\end{array}$ & $\begin{array}{l}400 \\
(10)\end{array}$ & $\begin{array}{l}128 \\
(21)\end{array}$ & $\begin{array}{l}423 \\
(23)\end{array}$ & 72 & 63 & 94 & 62 & 70 & 59 & 78 & 62 \\
\hline 449 & Lwówek Śląski & $\begin{array}{l}107 \\
(17)\end{array}$ & $\begin{array}{l}350^{\mathrm{s}} \\
(29)\end{array}$ & $\begin{array}{c}158 \\
(6)\end{array}$ & $\begin{array}{l}420 \\
(8)\end{array}$ & $\begin{array}{l}120 \\
(28)\end{array}$ & $\begin{array}{c}380 \\
(9)\end{array}$ & $\begin{array}{l}128 \\
(23)\end{array}$ & $\begin{array}{l}400 \\
(21)\end{array}$ & 65 & 56 & 90 & 68 & 86 & 75 & 80 & 66 \\
\hline 450 & Śnieżka & $\begin{array}{c}123 \\
(9)\end{array}$ & $\begin{array}{l}430^{f} \\
(24)\end{array}$ & $\begin{array}{l}165 \\
(10)\end{array}$ & $\begin{array}{l}490 \\
(16)\end{array}$ & $\begin{array}{l}148 \\
\text { (16) }\end{array}$ & $\begin{array}{l}450 \\
(23)\end{array}$ & $\begin{array}{l}145 \\
(17)\end{array}$ & $\begin{array}{l}470 \\
(23)\end{array}$ & 70 & 63 & 95 & 86 & 96 & 86 & 87 & 78 \\
\hline 466 & Jugów & $\begin{array}{l}119 \\
(4)\end{array}$ & $\begin{array}{l}400 \\
(15)\end{array}$ & $\begin{array}{l}167 \\
(6)\end{array}$ & $\begin{array}{c}480 \\
(6)\end{array}$ & $\begin{array}{l}136 \\
(14)\end{array}$ & $\begin{array}{l}410 \\
(25)\end{array}$ & $\begin{array}{l}141 \\
(16)\end{array}$ & $\begin{array}{l}430 \\
(24)\end{array}$ & 76 & 71 & 94 & 80 & 91 & 65 & 87 & 72 \\
\hline 487 & Bielsko & $\begin{array}{l}120 \\
(7)\end{array}$ & $\begin{array}{l}350^{s} \\
(26)\end{array}$ & $\begin{array}{l}162 \\
(6)\end{array}$ & $\begin{array}{c}420^{s} \\
(7)\end{array}$ & $\begin{array}{l}105 \\
(14)\end{array}$ & $\begin{array}{l}330^{s} \\
(10)\end{array}$ & $\begin{array}{l}129 \\
(20)\end{array}$ & $\begin{array}{l}367 \\
(21)\end{array}$ & 67 & 60 & 87 & 69 & 85 & 68 & 80 & 65 \\
\hline 488 & Bielsko & $\begin{array}{l}119 \\
(13)\end{array}$ & $\begin{array}{c}350^{s} \\
(9)\end{array}$ & $\begin{array}{l}157 \\
(11)\end{array}$ & $\begin{array}{l}430 \\
(11)\end{array}$ & $\begin{array}{c}86 \\
(28)\end{array}$ & $\begin{array}{l}320^{s} \\
(12)\end{array}$ & $\begin{array}{l}121 \\
(30)\end{array}$ & $\begin{array}{l}430 \\
(19)\end{array}$ & 76 & 70 & 86 & 40 & 96 & 54 & 86 & 54 \\
\hline 489 & Bielsko & $\begin{array}{l}131 \\
(7)\end{array}$ & $\begin{array}{l}390 \\
(25)\end{array}$ & $\begin{array}{l}163 \\
\text { (10) }\end{array}$ & $\begin{array}{c}420^{s} \\
(8)\end{array}$ & $\begin{array}{c}155 \\
(8)\end{array}$ & $\begin{array}{l}400 \\
(14)\end{array}$ & $\begin{array}{l}150 \\
(11)\end{array}$ & $\begin{array}{l}395 \\
(17)\end{array}$ & 67 & 62 & 94 & 88 & 92 & 65 & 84 & 72 \\
\hline 490 & Bielsko & $\begin{array}{l}118 \\
\text { (12) }\end{array}$ & $\begin{array}{l}400 \\
(15)\end{array}$ & $\begin{array}{l}172 \\
(14)\end{array}$ & $\begin{array}{l}450 \\
(19)\end{array}$ & $\begin{array}{l}142 \\
(16)\end{array}$ & $\begin{array}{l}430 \\
(7)\end{array}$ & $\begin{array}{l}144 \\
(19)\end{array}$ & $\begin{array}{l}427 \\
(21)\end{array}$ & 74 & 63 & 95 & 80 & 89 & 71 & 86 & 71 \\
\hline 491 & Bielsko & $\begin{array}{l}108 \\
(21)\end{array}$ & $\begin{array}{l}360 \\
(35)\end{array}$ & $\begin{array}{l}160 \\
(3)\end{array}$ & $\begin{array}{l}430 \\
(10)\end{array}$ & $\begin{array}{l}102 \\
(11)\end{array}$ & $\begin{array}{l}360 \\
(21)\end{array}$ & $\begin{array}{l}123 \\
(22)\end{array}$ & $\begin{array}{l}383 \\
(25)\end{array}$ & 62 & 54 & 93 & 44 & 96 & 54 & 84 & 50 \\
\hline 492 & Bielsko & $\begin{array}{l}121 \\
(8)\end{array}$ & $\begin{array}{l}400 \\
(23)\end{array}$ & $\begin{array}{l}155 \\
(8)\end{array}$ & $\begin{array}{c}410^{s} \\
(6)\end{array}$ & $\begin{array}{l}116 \\
(28)\end{array}$ & $\begin{array}{l}430 \\
(15)\end{array}$ & $\begin{array}{l}131 \\
(17)\end{array}$ & $\begin{array}{l}415 \\
(17)\end{array}$ & 65 & 57 & 89 & 63 & 68 & 62 & 74 & 60 \\
\hline 493 & Bielsko & $\begin{array}{l}122 \\
(17)\end{array}$ & $\begin{array}{l}400 \\
(24)\end{array}$ & $\begin{array}{l}157 \\
(2)\end{array}$ & $\begin{array}{l}420 \\
(15)\end{array}$ & $\begin{array}{l}138 \\
(19)\end{array}$ & $\begin{array}{l}431 \\
(22)\end{array}$ & $\begin{array}{l}139 \\
(16)\end{array}$ & $\begin{array}{l}417 \\
(20)\end{array}$ & 75 & 63 & 94 & 78 & 89 & 79 & 86 & 73 \\
\hline 494 & Bielsko & $\begin{array}{l}136 \\
(18)\end{array}$ & $\begin{array}{l}400 \\
(27)\end{array}$ & $\begin{array}{l}167 \\
(7)\end{array}$ & $\begin{array}{l}460 \\
(3)\end{array}$ & $\begin{array}{l}148 \\
(16)\end{array}$ & $\begin{array}{l}360^{s} \\
(13)\end{array}$ & $\begin{array}{l}150 \\
(14)\end{array}$ & $\begin{array}{l}430 \\
(19)\end{array}$ & 73 & 68 & 96 & 92 & 96 & 91 & 88 & 84 \\
\hline Multi-p & uation me & $\begin{array}{l}120 \\
(13)\end{array}$ & $\begin{array}{l}397 \\
(23)\end{array}$ & $\begin{array}{l}164 \\
(10)\end{array}$ & $\begin{array}{l}455 \\
(13)\end{array}$ & $\begin{array}{l}138 \\
(23)\end{array}$ & $\begin{array}{l}415 \\
(22)\end{array}$ & $\begin{array}{l}141 \\
\text { (19) }\end{array}$ & $\begin{array}{l}422 \\
(23)\end{array}$ & 69 & 60 & 93 & 78 & 90 & 72 & 84 & 70 \\
\hline
\end{tabular}


onto the $\mathrm{AE}$ axis describes stability - the higher the absolute value segment length, the lower the level of stability of the population. Therefore the GGE biplot represents the main effects and interactions attributable to the sites, while it cannot be used to determine the statistical significance of these effects.

Statistical analyses and presented biplots were performed with SAS/STAT 13.1 (SAS Institute, 2013) and specifically the GLM, MIXED, PRINCOMP and GPLOT procedures (Littell et al., 1996; Khattree \& Naik, 2000).

\section{Results}

The mean height of the beech trees across all the analysed environments in 2010 was $141 \mathrm{~cm}$ (Table 1). After five years of growth, the highest trees were those at the LZ site (mean $164 \mathrm{~cm}$ ), while the lowest (mean $120 \mathrm{~cm}$ ) were at the SB site. The same trend was still visible in 2015, when the mean height across all the environments was $422 \mathrm{~cm}$. Then the tallest trees were still at the LZ site (mean $455 \mathrm{~cm}$ ), and the shortest were at the SB site (mean $397 \mathrm{~cm}$ ). In Sucha Beskidzka (SB) the fastest height growth was found for provenances 378, 392, 420, 431 and 450 , and the most limited height growth was found for populations 430, 438, 449, 487 and 488. In LZ, the fast-growing trees were from populations 378 , $392,424,437$ and 446 , while slow growth was noted for trees from populations 430, 434, 487, 489 and 492. At the ZL site, fast growth on height was noted for the progeny of populations 377, 392, 420, 431 and 439 , while the slow-growing trees there were from populations 430, 438, 487, 488 and 494.

Trees of population 392 proved capable of rapid growth on height irrespective of environmental conditions. In turn, the progeny of three populations $(378,420$ and 431$)$ showed good growth at two of the three sites, while the weakest growth at all sites characterised offspring of population 430, 438 and 487.

Statistically significant effects of populations, environment and $\mathrm{G} \times \mathrm{E}$ interaction were found for tree height in 2010 and 2015 (Table 2). According to the decomposition of variance (DOV) in 2010, $51.6 \%$ of the overall variation in height $(\mathrm{G}+\mathrm{E}+\mathrm{GE})$ could be accounted for by the effect of environment, while the remaining $48.4 \%$ was explained in terms of the impact of genotype, $\mathrm{G}(25.6 \%)$, and $\mathrm{G} \times \mathrm{E}$ interaction (22.9\%).

According to DOV in $2015,63.1 \%$ of the overall variation in tree height $(\mathrm{G}+\mathrm{E}+\mathrm{GE})$ was explicable in terms of the impact of $\mathrm{E}$, leaving $36.9 \%$, of which the effect of G, accounted for $17.9 \%$ of the variation, and the effect of the GXE interaction for $18.9 \%$. Throughout the 2010-2015 period between measurements, the effect on height of beech trees attributable to the environment increased by $11.5 \%$, while the influence of genotype and the GXE interaction was lower by $7.6 \%$ and $3.9 \%$, respectively.

To determine the changes in tree height in the studied environments, the PCA was carried out separately for 2010 and 2015. In the case of the 2010 data, the division of effects $(G+G E)$ with the aid of the GGE biplot method yielded the result that PC1 explained $82.6 \%$ of the variation $(\mathrm{G}+\mathrm{GE})$, while the second component PC2 explained a further $12.2 \%$. In the case of the 2015 data, the division of effects $(\mathrm{G}+\mathrm{GE})$ with the aid of the GGE biplot yielded the result that $\mathrm{PC} 1$ explained $67.9 \%$ of the variation $(G+G E)$, while PC2 explained a further $20.9 \%$.

\section{Environmental similarity and production potential}

The positioning of the environments in relation to the $\mathrm{AE}$ axis shows that both in 2010 and 2015 trees were tallest at the LZ study site (Fig. 2). In turn, the SB site had an environment that was the most similar to the average environment (AE), as was revealed in both tree heights as such, and the stability of their values. The ZL site had trees characterised by the greatest instability of measured heights, thus it contributed more to the $\mathrm{G} \times \mathrm{E}$ interaction in terms of tree heights than the other two sites.

Comparison of the positions of the environments between two ages in Fig. 2 reveals a slowing growth of trees at the SB site during the 2010-2015 period.

Table 2. Results of the analysis of variance for tree height in 2010 and 2015 among 30 populations of beech in three environments

\begin{tabular}{|c|c|c|c|c|c|c|c|c|c|c|}
\hline \multirow[b]{2}{*}{ Source of variation } & \multicolumn{5}{|c|}{2010} & \multicolumn{5}{|c|}{2015} \\
\hline & $\mathrm{Df}$ & MS & $\mathrm{F}$ & $\mathrm{P}$ & $(G+E+G E)$ & $\mathrm{Df}$ & MS & $\mathrm{F}$ & $\mathrm{P}$ & $(\mathrm{G}+\mathrm{E}+\mathrm{GE})$ \\
\hline Genotype (G) & 29 & 1467.83 & $2.24^{1}$ & 0.005 & 25.58 & 29 & 10426 & $1.90^{1}$ & 0.019 & 17.98 \\
\hline Environment (E) & 2 & 42897 & $8.5^{1}$ & 0.006 & 51.56 & 2 & 530599 & $7.72^{1}$ & 0.010 & 63.1 \\
\hline Block (Environment) & 9 & 4625.72 & 19.91 & $<0.001$ & - & 9 & 66536 & 20.27 & $<0.001$ & - \\
\hline Genotype $\times$ Environment $(\mathrm{G} \times \mathrm{E})$ & 58 & 655.99 & 2.82 & $<0.001$ & 22.86 & 58 & 5488.58 & 1.67 & 0.004 & 18.92 \\
\hline Experimental error & 261 & 232.37 & - & - & - & 261 & 3282.51 & - & - & - \\
\hline
\end{tabular}

${ }^{1}$ Hocking's approach.

Df - degree of freedom, MS - mean squares, F - statistic, P - value. 

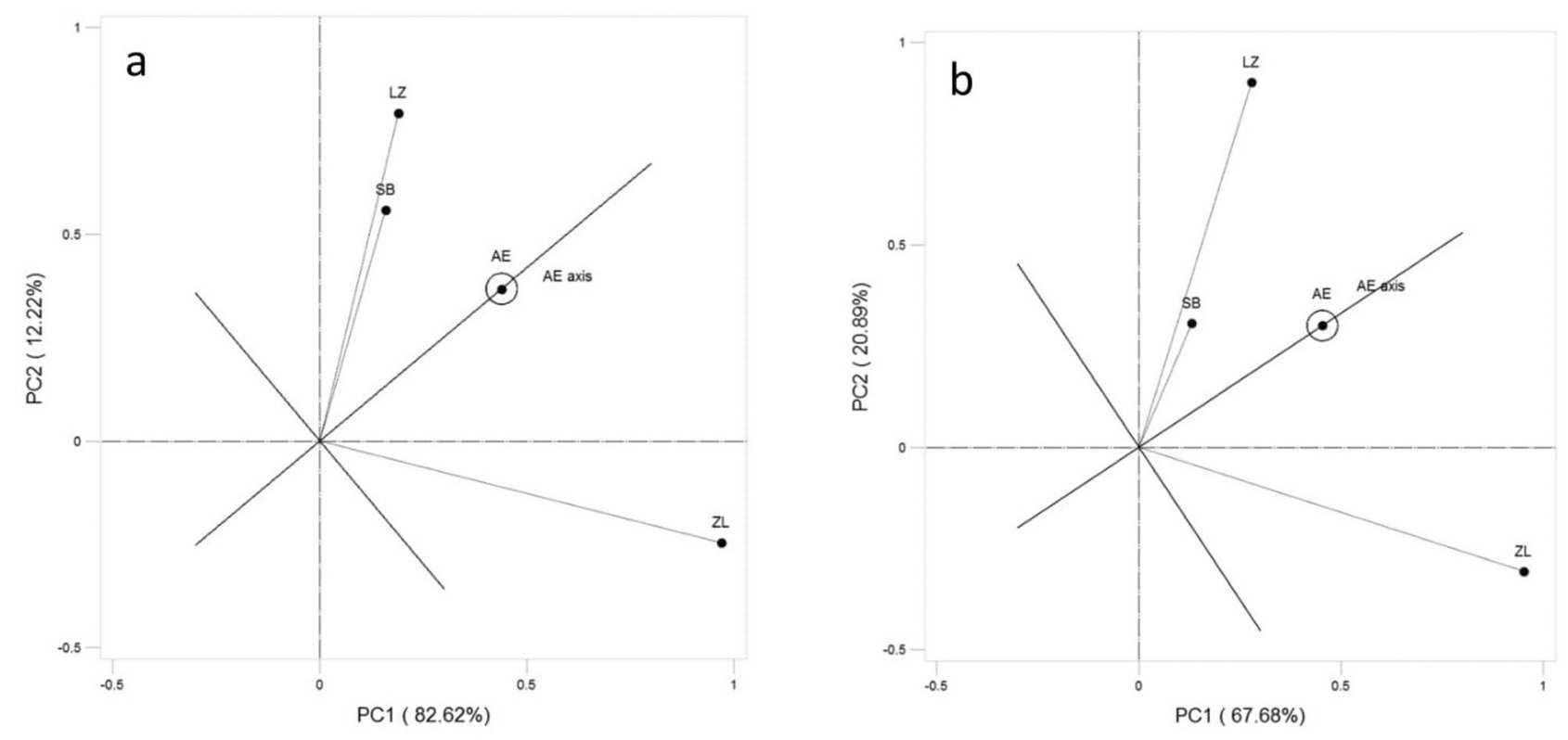

Fig. 2. Production potential and the similarity of studied environments in relation to the average environment (AE) as of 2010 (a) and 2015 (b)

Table 3. Correlation coefficients (above diagonal) and $\alpha_{i j}$ angles (below diagonal) between pairs of environments (upper value in cell - 2010, lower value - 2015; ** significant at the 0.01 probability level, ns - not significant)

\begin{tabular}{cccc}
\hline Environments & LZ & SB & \multicolumn{1}{c}{ ZL } \\
\hline LZ & - & $0.99^{* *}$ & $-0.01 \mathrm{~ns}$ \\
& & $0.99^{* *}$ & $-0.01 \mathrm{~ns}$ \\
SB & 2.38 & & $0.03 \mathrm{~ns}$ \\
& 5.91 & - & $0.09 \mathrm{~ns}$ \\
ZL & 90.80 & 88.42 & - \\
& 90.65 & 88.73 & - \\
\hline
\end{tabular}

In contrast, while characterised by unstable increases in the heights of beech trees, the ZL site was found to differ from SB in its greater growth increments over the 2010-2015 period. The increments characterising trees at the LZ site were at an average level.

Analysis of the angles between the vectors for the environments revealed that the LZ and SB sites were most similar to each other both in 2010 and 2015 (Fig. 2). The vector for the ZL site created a nearright angle with the vectors for the two remaining environments, which indicates that correlation coefficients between the ZL and the LZ and SB sites were non-significant (Table 3).

\section{Similarity and stability of populations}

The type of biplot as presented at fig. 3 allows to analyse similarity/dissimilarity among the experimental sites and to identify populations adapted to specific environments. The 30 populations of beech and 3 environment (each studied in 2010 and 2015) were distributed across 8 areas (Fig.3). The
ZL and LZ sites were very different from each other, while the SB site was similar to the LZ site. This indicated the existence of two mega-environments: LZ+SB (ME1) versus ZL (ME2). Populations specifically adapted to the conditions of ME1 were 446, 432 (good and stable growth) and 437 (good growth but less stable). Specifically adapted to the ME2 (ZL) were populations 420 and 431, for which the height increment was the largest in this area (Fig. 3).

Progeny of populations $378,439,392,377$ and 450 grew fastest when averaged across all locations (Fig. 3; Tab. 1). These populations (specifically and widely adapted) should be recommended for future use. On the other hand, progeny of populations 488,487 , 438, 430, 449 and 491 performed poorly in terms of three height at all experimental sites.

The lengths of vectors for the environments are an indication of their discrimination ability of populations (Fig. 3). The environments in 2010 had very short vectors. This is understandable, because tree heights in 2010 did not differ among the populations as much as in 2015. Environment ZL in 2015 was most discriminating as indicated by its long vector.

On the GGE biplot, stability of a population is described by reference to its position along the $\mathrm{AE}$ axis (Fig. 4). The closer to $\mathrm{AE}$ axis populations are plotted, the more stable they are (i.e. showing small interaction with environments). Among the stable populations the most interesting are those with fast growth in all environments (highest values of $\mathrm{AE}$ axis). The populations $378,439,392,377$ and 450 emerged as stable and tall (Fig. 4). Populations 446, 437, 432 and 424 were also tall, but less stable.

The GGE biplot based on differences between 2015 and 2010 data was used to describe changes 


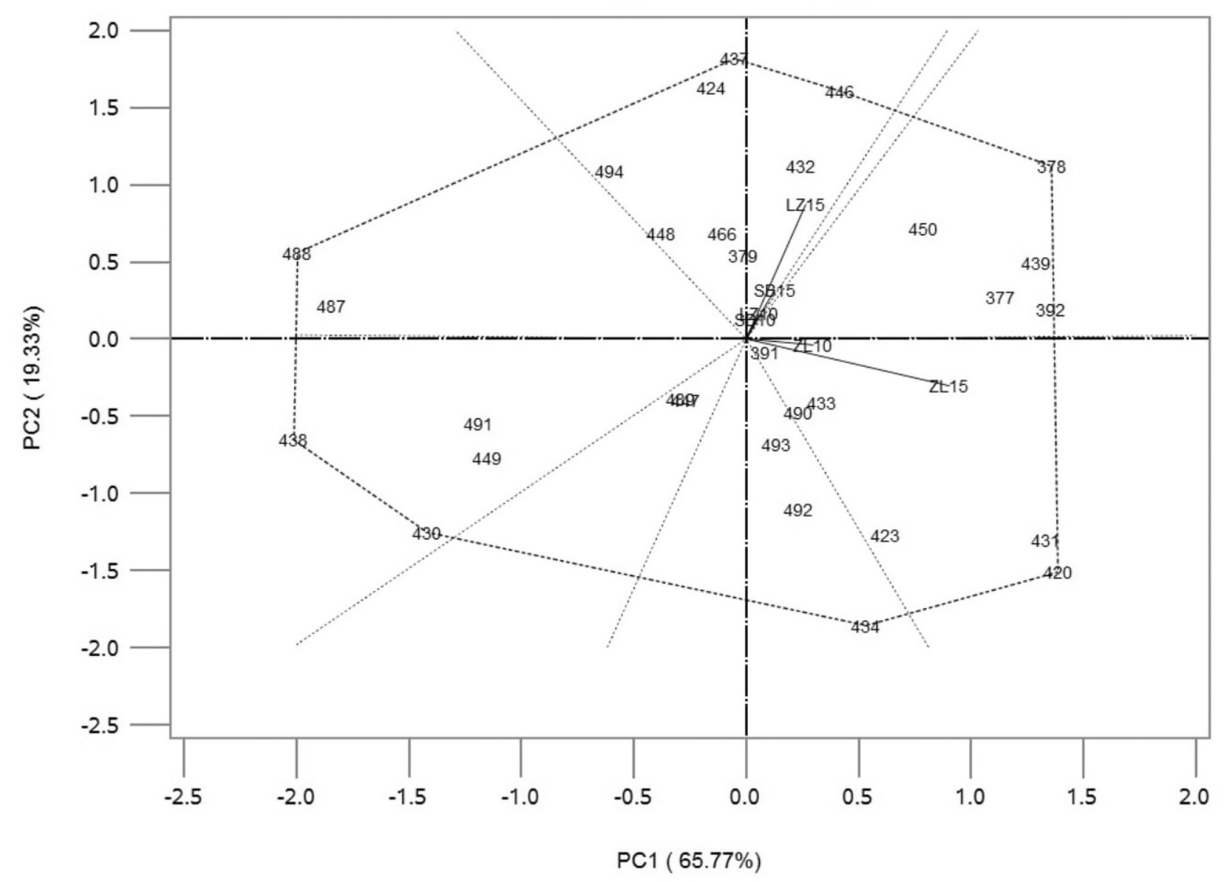

Fig. 3. A polygon superimposed on the GGE biplot on the basis of the first two PCs for 30 beech populations and three environments in the years 2010 and 2015

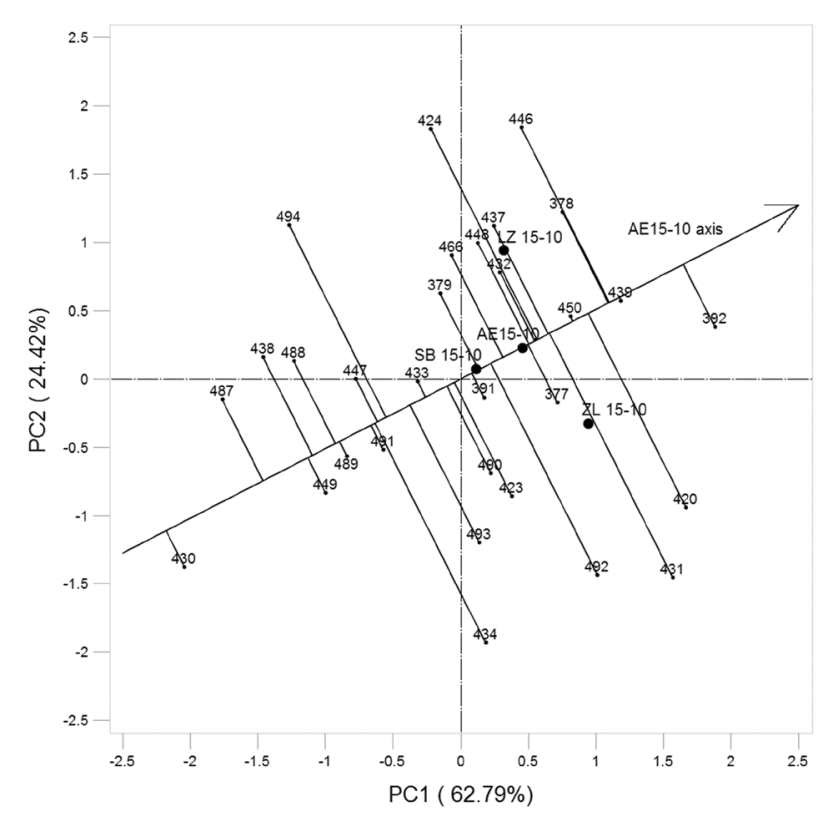

Fig. 4. The GGE biplot of mean height and its stability in relation to the average environment (AE) for 30 European beech populations

in the height growth and its stability across environments and years (Fig.5). Orthogonal projection of population on the AE15-10 axis indicated difference between average height in 2015 in comparison to 2010, and an arrow on the AE15-10 axis indicates direction of these differences. Populations for which tree height differences between 2010 and 2015 were stable across all environments are located closest to

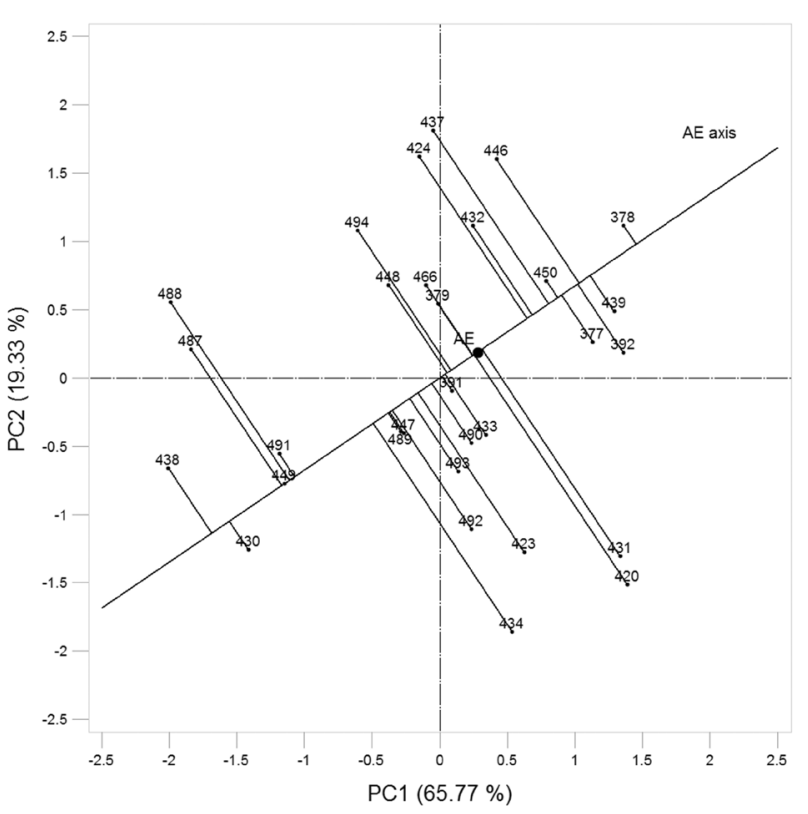

Fig. 5. Changes in the height growth and its stability for the studied beech populations between 2010 and 2015 .

the AE15-10 axis (Fig. 5). Populations 392, 439 and 378 grew fastest and stable during analysed growing period across all sites (Fig. 5). Progeny of population 446 was specifically good for the LZ environment while 420 and 431 grew fast in ZL. The fastest growing progenies for the SB site originated form populations 439 and 450. 


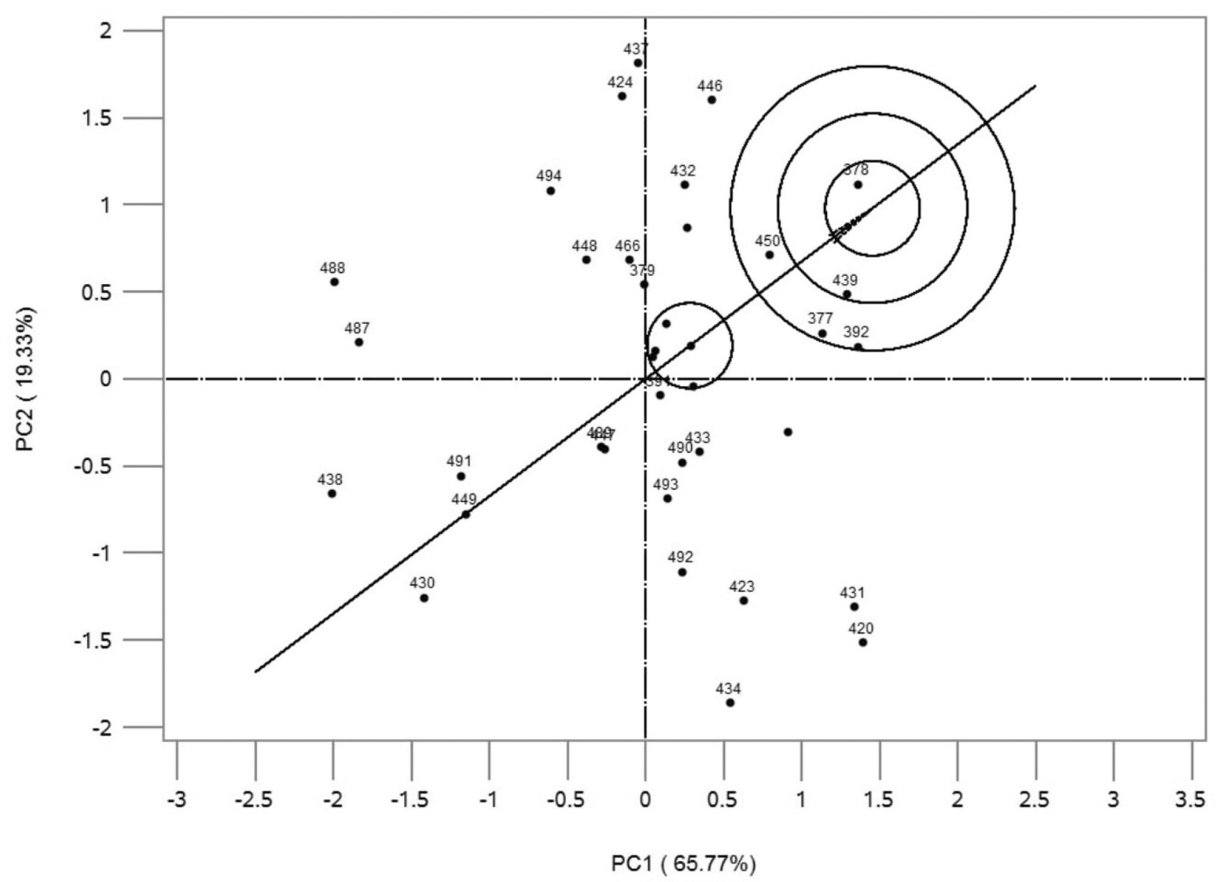

Fig. 6. Comparison between the analysed populations and the theoretical "ideal population"

\section{The "ideal population"}

The "ideal population" would be characterised by a high average value for height increments, with values for such trees growing in different environments also displaying a high degree of stability. The "ideal population" is determined by the vector beginning at the center of the coordinate system and ending at the orthogonal projection on the $\mathrm{AE}$ axis of the population which has the greatest PC1 value (Fig. 6). Simultaneously the "ideal population" is absolutely stable as as a result of the location on the $\mathrm{AE}$ axis (zero value for the $\mathrm{G} \times \mathrm{E}$ interaction in the average environment) (Yan \& Kang, 2002).

For easier discovery of objects positioned closest to the end of the vector, the circles centred on that point were drawn. The first such circle includes population 378, which represented the ideal population in the sense that large increments in the heights of beech trees were noted, along with a high degree of stability for these values across environments. The second circle includes the population 439 , for which height increments were smaller, but degree of stability was high.

\section{Discussion}

The multiannual nature of development of woody plants and the highly dynamic growth with low stability during a juvenile phase likely determine the relatively rare use of the biplot graphic method in breeding experiments with trees. Nevertheless, interest in this method has grown in recent years (Zhao et al., 2016; Klisz et al., 2017). This can be linked to a broad scope of possibilities for interpretation in the case of data obtained from multi-enviroment genetic experiments. An assessment of variability explained by the effects of $\mathrm{G} \times \mathrm{E}$ interaction allows to determine the scale of reduction in genetic gain achievable through selection of genotypes (Murillo, 2001). Furthermore, calculation of the GXE effect offers conclusions regarding allowable transfers of seeds, and the associated designation of seed regions for tested genotypes and populations (Taibi, 2014). The identification of the most favourable origins from the point of view of an analysed trait or group of traits allows to narrow down the selection to genotypes promotable in the plantations grown over short production cycles (Ding et al., 2008; Sixto et al., 2015). The use of biplots in interpreting multi-environment experiments with narrowed selection criteria also allows above-average genotypes to be identified (Zhao et al., 2016). In turn, where genotypes specifically adapted to selected test environments, and stable in the sense of manifesting favorable values for the analysed traits are distinguished, it becomes possible to formulate guideline strategies for afforestation and selective breeding, as well as the protection of gene resources (Correia et al., 2010).

The GGE biplot method used in stability testing for crop yields can also be used successfully in stability analysis concerning breeding of forest trees. To find an environment in which genotypes can be assessed effectively, use is made of a GGE biplot with an AE axis (Fig. 2). The selected environment should 
be one that both distinguishes effectively between the populations and is representative of the target region (Yan, 2002). In our studies, we identified two mega-environments in which growth conditions were similar to each other in both 2010 and 2015. Environments LZ and SB were more representative than environment $\mathrm{ZL}$, while the latter achieved the most effective discrimination of populations. The biplot (Fig. 3) shows the discrimination power of the environments as indicated by the vector length of the environments. Apparently the differences among populations in 2015 were much larger than those in 2010. Therefore, the 2015 data are more useful than the 2010 data in population evaluation. However, the biplot also shows the 2010 data and the 2015 data were highly correlated within locations (Tab. 3), therefore, the 2010 data are also useful if 2015 data were not available. This means that effective evaluation of populations could be conducted 5 years earlier, although the 2015 data are more reliable.

The results of our research showed that local populations did not prove to be better than others in terms of height growth in any of the analysed environments. Population 438 which is local to the SB site showed the most limited height increments there. Similar behaviour was displayed by the population 431, which is local to the LZ environment (Fig. 3). Earlier results at experimental plots in ZL and LZ point to the need for particular populations, though not necessarily local ones, to be chosen for cultivation at a given site (Barzdajn, 2009). Similar conclusions were reached by Buraczyk et al. (2016), after analysis of various quantitative and qualitative traits among 23 progenies of European beech in southeastern Poland.

Better adaption of the non-local populations seems to be particularly important in the context of the counteracting unfavourable influence of climate change on European beech populations in Poland. Because rate of the climate change is likely to be faster than evolutionary adaptiveness (Aitken et al., 2008; Alberto et al., 2013), the use of assisted migration and seed transfer seems to be promising breeding activity (Bolte et al., 2016).

In our analysis we found a significant effect of genotype on differences in tree height. However, this influence of genotype declined through time. Our results contradict those found for the growth of beech in central Poland (Szeligowski, 2012). In that study analysing the height of beech trees from 47 Polish populations, the percentage input due to genotype was greatest at age 15 years (at $46.3 \%$ ), while it was most limited in the case of 9-year-old trees (at $32.1 \%$ ). In our study the overall variability conditioned by a genotype was greater at age of 5 years (a $25.6 \%$ input), and went down to $17.9 \%$ by the age of 10 years. This shows at the same time a greater influence of the environment on height of trees. This environmental influence grew steadily with increasing age from $51.6 \%$ in 2010 to $63.1 \%$ in 2015 .

Of considerable interest are the results concerning changes in the mean height increments between the years 2010-2015 (Fig. 5). During this period the populations showed varied reactions from the height-increment point of view. For some populations the tree height increment increased (as in the case of 392 and 378 ) or decreased (430, 487 and 449) between two measurement occasions. There were also those (like 439 and 450) maintaining growth at very stabilised levels across all three environments.

There are two possible approaches to using the obtained results in further silvicultural work with the analysed populations of European beech in this testing region. The first approach, described by Finlay and Wilkinson (1963), assumes a search for stable populations, followed by their wide utilisation in different environments. Looking at our results in line with this approach, we report that populations like 378, 439, 446 and 392 and stand out from the set of studied populations. They do not manifest $\mathrm{G} \times \mathrm{E}$ interactions and reach large height on all three analysed plots through the whole period of investigation. For these reasons the populations in question can be considered worthy of wide introduction into forest management. Their high value is further confirmed by their similarity to the "ideal population" (Fig. 6). In contrast, population 430 shows stability of tree height but is one of the populations manifesting most limited growth. Populations 488 and 438 grow as weakly as 430 , but in their case the trait was less stable. These three populations should thus be excluded from further use in the region were testing was conducted.

A second approach, (e.g.McKeand et al., 1990; Baltunis et al., 2010) assumes the delineation of breeding zones in which specifically-adapted populations are used. In our work, we confirmed the presence of such populations, which are suitable for recommendation in breeding in conditions only represented by a defined study plot. The three study sites formed two mega-environments in which the introduction of specifically adapted populations can be advocated. In the case of the ZL site these were populations 420, 431 and 423, whereas for the SB and LZ sites these were populations 437, 424, 432 and 446. Populations adapted to local conditions and stable across the different environments were also indentified in the work of Stojnić et al. (2015), who assessed tree height and survival at 5 and 6 years after planting among 15 beech populations in four areas of Bosnia-Herzegovina, Croatia and Serbia. In our network of progeny testing the tested populations are compared with different types of standards of national, regional or local range. These are most 
often populations of known genetic value established on the basis of earlier research. In our study these were populations 447, 391 and 392 that were selected for testing as the regional standards for the region III. The obtained results show that only one of those standards was selected effectively (391). On the GGE biplot (Fig. 4) the population 391 was located close to the centre of the coordinate system, denoting that it was characterised by average height and stability of the trait. Furthermore 391 manifested no changes in height increments between 2010 and 2015 (Fig. 5). The other two populations selected as the regional standards showed contrasting growth pattern. According to Giertych (1990; 2000), European beech shows mostly the ecotypic variation, with each population associated with its habitat. This accounts for a lack of economic benefit if seeds from a distant population are utilised. Under these circumstances, the value of a population will depend on the similarity, or the lack thereof, of environmental conditions between deployment zone and the area of the parental population. Within the analysed set of populations it is possible to distinguish (unstable) ones that were characterised by above-average mean heights in given environments only. Equally, there were populations average as regards tree height that were nevertheless stable across different environments representing varied conditions for growth. This same kind of ranking of genotypes in terms of their stability and the mean value for a given trait was presented in studies of Betula platyphylla by Zhao et al. (2016).

Progeny testing in the last 10 years has shown that conclusions as regards to specific genotype adaptation may be encumbered with errors. These reflect the negative effect of a potentially discriminatory environment. It must be emphasized that differences in height growth and survival after 5 or 10 years do not fully describe adaptation potential of European beech. On the other hand, early developmental stages of plants are expected to be more sensitive to climate change than adult stages (Walck, 2011). On the basis of our results we cannot determine a geographical pattern for the populations of beech that grow best in terms of height. Populations with the most stable values for height in all three environments can be found in both the mountain and the upland region. It is possible to identify populations that adapt well to local conditions, as well as to growing conditions in the whole testing region. The GGE biplot method used in stability testing for crop yields can also be used successfully in stability studies involving the growth of forest trees.

\section{Acknowledgments}

The study was funded by the State Forests National Forest Holding, as part of research project BLP-375.
We acknowledge comments by two anonymous reviewers and the associate editor, which helped to improve the earlier versions of the manuscript.

\section{References}

Aitken SN, Yeaman S, Holliday JA, Wang T \& Curtis-McLane S (2008) Adaptation, migration or extirpation: climate change outcomes for tree populations. Evolutionary Applications 1: 95-111. doi:10.1111/j.1752-4571.2007.00013.x.

Alberto FJ, Aitken SN, Alía R, González-Martínez SC, Hänninen H, Kremer A, Lefèvre F, Lenormand T, Yeaman S, Whetten R \& Savolainen O (2013) Potential for evolutionary responses to climate change - evidence from tree populations. Global Change Biology 19: 1645-1661. doi:org/10.1111/ gcb.12181.

Annicchiarico P (2002) Genotype x environment interactions. Challenges and opportunities for plant breeding and cultivar recommendations. FAO, Rome. Plant Production and Protection Paper 174.

Baltunis BS, Gapare WJ \& Wu HX (2010) Genetic parameters and genotype by environment interaction in Radiata pine for growth and wood quality traits in Australia. Silvae Genetica 59: 113-124.

Barzdajn W (2009) Adaptacja i początkowy wzrost potomstwa drzewostanów nasiennych buka zwyczajnego (Fgus sylvatica L.) na uprawach porównawczych $\mathrm{w}$ nadleśnictwach Złotoryja i Lądek Zdrój. Leśne Prace Badawcze 70: 101-111.

Bolte A, Czajkowski T, Cocozza C, Tognetti R, de Miguel M, Pšidová E, Ditmarová 'L, Dinca L, Delzon S, Cochard $\mathrm{H}$, Ræbild A, de Luis $\mathrm{M}$, Cvjetkovic B, Heiri C \& Müller J (2016) Desiccation and Mortality Dynamics in Seedlings of Different European Beech (Fagus sylvatica L.) Populations under Extreme Drought Conditions. Frontiers in Plant Science 7: 751. doi:10.3389/ fpls.2016.00751.

Boratyńska K \& Boratyński A (1990) Systematyka i geograficzne rozmieszczenie: Buk zwyczajny Fagus syvatica L. Nasze drzewa leśne (ed. by S Białobok) Monografie Popularnonaukowe. PWN, Warszawa - Poznań, pp. 27-73.

Buraczyk W, Szeligowski H, Studnicki M, Drozdowski S \& Bielak K (2016) Multivariable evaluation of the early growth performance of European beech (Fagus sylvatica L.) provenances from south-eastern Poland. Sylwan 160: 981-992.

Correia I, Al'ia R, Yan W, David T, Aguiar A \& Almeida MH (2010) Genotype $\times$ Environment interactions in Pinus pinaster at age 10 in a multi-environment trial in Portugal: a maximum likelihood approach. Annals of Forest Science 67: 612. doi:10.1051/forest/2010025. 
Crossa J (1990) Statistical analyses of multilocation trials. Advances in Agronomy 44: 55-85. doi:10.1016/S0065-2113(08)60818-4.

DeLacy IH, Basford KE, Cooper M \& Bull JK (1996) Analysis of multi-environment trials - an historical perspective. Plant adaptation and crop improvement (ed. by M Cooper \& GL Hammer) CAB International, pp. 39-124.

Ding M, Tier B, Yan W, Wu HX, Powell MB \& McRae TA (2008) Application of GGE biplot analysis to evaluate Genotype (G), Environment (E), and $\mathrm{G} \times \mathrm{E}$ interaction on Pinus radiata: a case study. New Zealand Journal of Forestry Science 38: 132142.

Dzwonko Z (1990) Ekologia: Buk zwyczajny Fagus syvatica L. Nasze drzewa leśne (ed. by S Białobok) Monografie Popularnonaukowe. PWN, Warszawa - Poznań, pp. 27-73.

Finlay KW \& Wilkinson GN (1963) The analysis of adaptation in a plant breeding programme. Australian Journal of Agricultural Research 14: 742 754. doi:10.1071/AR9630742.

Fonder W, Matras J \& Załęski A (2007) Leśna baza nasienna w Polsce. CILP, Warszawa.

Gauch GH \& Zobel RW (1997) Identifying mega-environments and targeting genotypes. Crop Science 37: 311-326.

Giertych M (1982) Breeding strategies with Norway spruce in Poland. Proc. of the IUFRO joint meeting of working parties on genetics about breeding strategies incl. multi-clonal varietes. Sensenstein, pp. 17-21.

Giertych M (1990) Genetyka: Buk zwyczajny (Fagus sylvatica L.) Nasze drzewa leśne (ed. by S Białobok) Monografie Popularnonaukowe, PWN, Warszawa - Poznań, pp. 193-236.

Giertych M (2000) Zmienność genetyczna buka. Zeszyty Naukowe Akademii Rolniczej im. H. Kołłątaja w Krakowie 69: 35-45.

Golub GH \& Reinsch C (1971) The singular value decomposition and least squares solutions. Handbook for Automatic Computation (ed. by JH Wilkinson \& C Renisch) Springer-Verlag Berlin, pp. 134-151.

Hocking RR \& Speed FM (1975) A full-rank analysis of some linear model problems. Journal of the American Statistical Association 70: 706-712.

Ivanković M, Bogdan S \& Božič G (2008) Varijabilnost visinskog rasta obične bukve (Fagus sylvatica L.) $\mathrm{u}$ testovima provenijencija $\mathrm{u}$ Hrvatskoj I Sloveniji. Šumarski list 11/12: 529-541.

Kang MS (1990) Understanding and utilization of genotype-by-environment interaction in plant breeding: Genotype-by-environment interaction and plant breeding (ed. by MS Kang) Baton Rouge, Louisiana State University Agricultural Center, pp. 52-68.
Kang MS \& Gauch GH (1996) Genotype-by-environment interaction. CRC Press, Boca Raton, FL.

Khattree R \& Naik DN (2000) Multivariate data reduction and discrimination with SAS software. SAS Institute Inc., Cary, NC.

Klisz M, Jastrzębowski Sz, Ukalski K, Ukalska J \& Przybylski P (2017) Adaptation of Norway spruce populations in Europe: A case study from northern Poland. New Zealand Journal of Forestry Science 47: 8. doi:10.1186/s40490-017-0090-6.

Krahl-Urban J (1958) Vorläuftige Ergebnisse von Buchen Provenienzversuchen. Allgemeine Forst-und Jagdzeitung 129: 245-251.

Littell RC, Milliken GA, Stroup WW \& Wolfinger RD (1996) SAS system for mixed models. SAS Institute Inc., Cary, NC.

McKeand SE, Li B, Hatcher AV \& Wei RJ (1990) Stability parameter estimates for stem volume for loblolly pine families growing in different regions in the southeastern United States. Forest Science 36: 10-17.

Murillo O (2001) Genotype by environment interaction and genetic gain on unbalanced Pinus oocarpa provenances trials. Agronomia Costarricense 25: $21-32$.

Piepho HP (1997) Analyzing genotype-environment data by mixed models with multiplicative terms. Biometrics 53: 761-766.

Raport o lasach (2014) CILP, Warszawa.

Rodrigues PC, Mejza S \& Mexia JT (2008) Structuring genotype $\times$ environment interaction - an overview. Biuletyn Instytutu Hodowli i Aklimatyzacji Roślin 250: 41-57.

Sabor J (1999) Kierunki badań genetycznych drzew leśnych w doświadczalnictwie leśnym: Stan i perspektywy badań z zakresu hodowli lasu (ed. by A Gorzelak \& T Zachara) Materiały I Konferencji Leśnej, Sękocin Las, 18 - 19 maja 1999, Warszawa, pp. 133-138.

Saxton AM (2004) Genetic analysis of complex traits using SAS. SAS Institute, Inc. Cary, N.C.

SAS Institute Inc (2013) SAS/STAT 13.1 User's Guide. SAS Institute Inc., Cary, North Carolina.

Sixto H, Gil PM, Ciria P, Camps F, Cañellas I \& Voltas J (2015) Interpreting genotype-by-environment interaction for biomass production in hybrid poplars under short-rotation coppice in Mediterranean environments. GCB Bioenergy 8: 1124-1135. doi:10.1111/gcbb.12313.

Stojnić S, Orlović S, Ballian D, Ivanković $M$, Šijačić-Nikolić M, Pilipowić A, Bogdan S, Kvesić S, Mataruga M, Daničić V, Cvejtković B, Miljković D \& von Wuehlisch G (2015) Provenance by site interaction and stability analysis of European beech (Fagus sylvatica L.) provenances grown in common garden experiments. Silvae Genetica 64: 133-147. 
Szeligowski H (2012) Zmienność oraz wartość hodowlana populacji buka zwyczajnego $\mathrm{W}$ warunkach siedliskowych centralnej Polski. Wydawnictwo SGGW, Warszawa.

Taibi K (2014) Integrated approach for addressing assisted population migration programs in forest management to climate change: out-planting performance, genotype by environment interactions, physiological and molecular response. Dissertation. The Polytechnic University of Valencia, Spain.

Tarasiuk S (1992) Recent anthropogenous distribution of European Beech outside its natural Range in Pland. Folia Forestalia Polonica Series A-Forestry 34: 31-38.

Teissier du Cros E (1977) Étude da la viaribilité du hêtre. Revue Forestière Française 29: 355-362.

Ukalski K, Śmiałowski T \& Ukalska J (2010a) Analysis of oat yield environments using graphical GGE method. Colloquium Biometricum 40: 81-93.

Ukalski K, Śmiałowski T \& Ukalska J (2010b) Analiza plonowania i stabilności genotypów owsa za pomocą metody graficznej typu GGE. Żywność. Nauka. Technologia. Jakość. 3: 127-140.

Ukalski K \& Klisz M (2016) Application of GGE biplot graphs in multi-environment trials on selection of forest trees. Folia Forestalia Polonica Series A-Forestry 58: 228-239. doi:10.1515/ffp2016-0026.

Walck JL, Hidayati SN, Dixon KW, Thompson K \& Poschlod P (2011) Climate change and plant regeneration from seed. Global Change Biology 17: 21452161. doi:10.1111/j.1365-2486.2010.02368.x.

Wüehlisch von G (2004) Series of international provenance trials of European beech: Improvement and silviculture of beech. Proceedings from the $7^{\text {th }}$ international beech symposium IUFRO research group 1.10.00, 10 - 20 May 2004, Teheren, Iran, pp. 135-144.

Wüehlisch von G, Hansen JK, Mertens P, Lesebach M, Meierjohann E, Muhs HJ, Teissier du Cros E \& de Vries S (2008) Variation among Fagus sylvatica and Fagus orientalis provenances in young international field trials: Proceedings the $8^{\text {th }}$ IUFRO International Beech Symposium, 8 - 13 September 2008, Nanae, Hokkaido, Japan, pp. 25-27.

Yan W, Hunt LA, Sheng Q \& Szlavnics Z (2000) Cultivar evaluation and mega-environment investigation based on the GGE biplot. Crop Science 40: 597-605. doi:10.2135/cropsci2000.403597x.

Yan W \& Hunt LA (2001) Interpretation of genotype $\mathrm{x}$ environment interaction for winter wheat yield in Ontario. Crop Science 41: 19-25. doi:10.2135/ cropsci2001.41119x.

Yan W, Cornelius PL, Crossa J \& Hunt LA (2001) Two types of GGE biplots for analyzing multienvironment trial data. Crop Science 41: 656-663. doi:10.2135/cropsci2001.413656x.

Yan W \& Rajcan I (2002) Biplot analysis of test sites and trait relations of soybean in Ontario. Crop Science 42: 11-20. doi:10.2135/cropsci2002.1100.

Yan W \& Kang MS (2002) GGE biplot analysis: a graphical tool for breeders, genetics and agronomists. CRC Press, Boca Raton, FL.

Yeiser JL, Lowe W \& van Buijtenen JP (2001) Stability and seed movement for loblolly pine in the Western Gulf Region. Silvae Genetica 50: 81-88.

Zhao X, Xia H, Wang X, Wang C, Liang D \& Li K (2016) Variance and stability analyses of growth characters in half-sib Betula platyphylla families at three different sites in China. Euphytica 208: 173-186. doi:10.1007/s10681-015-1617-7. 